\title{
Perceptual design method research in product design
}

\author{
Wenming Liu ${ }^{1}$, Hao $\mathrm{Cui}^{1 *}$ \\ Shenyang Jianzhu University, Shenyang, Liaoning, 110168, China
}

\begin{abstract}
On the purpose of deepening method research of perceptual analysis, based on the description of the relationship between perceptual design and rational design, the importance of perceptual design in modern product design is clarified. To strengthen the emotional expression of the product, the study focuses on sort perceptual design method with the background of perceptual design internalization and equivalent theory. Taking the perceptual design as the bond, how the method located sensibility in product design is analyzed. By taking advantage of formal beauty rule, more perceptual elements are added into product design to summarize the method and principle based on the product perceptual design.
\end{abstract}

\section{Research background of perceptual design}

In terms of the definition of perception, perception refers to people's subjective feeling to things, which is affected by complicated psychological elements. After getting the external motive, the receptor got the import and finished a series of procession, then it can generalize the final subjective feeling, that is, the result generalized by the mutual effect between products and people. The perception belongs to the transition zone between rationality and irrationality, which is always used to balance the contradict between them.

On the basis of the product functional design as well as exterior design, perceptual design easily converts design content into acquired information to get resonance with consumers, affecting people's emotions indirectly. At the same time, perceptual design improves user's sense of participation. In designing products, it is a vital way to combine humanized design, emotional design and other functions, which is an important means of user-centered design.

\subsection{Research purposes of perceptual design}

On the one hand, urgent perceptually request and the research to receptor function, not only provides a new design view and design channel, but also widens the area which can be explored by perceptional product design. In other words, it aims to satisfy people's requests for a better life, emphasizing more the closer perceptual relationship between users and products under the new background at the time. On the other hand, more and more requests requiring product and technology enjoy the same quality. Embedding perceptual product design concept into the development and design of business product can make firms more competitive in the complex market. Mutually, the develop process of perceptual product design also contributes to scientific perceptual design.

\subsection{Development status of perceptual design}

Browsing the international trend, Japan and other western countries established more complete perceptual design system, while China is still at the initial stage of development. For our own country, perceptual depends much on designer's exploration about product's exterior. After acquiring skills, including accumulated knowledge, summarized culture, technology survey etc., they can finish the innovative creature on the basis of above skills. This method shows the designers' high request for basic training. The lack of more systematical theory basis as the design resource is also the reason for the weakness of domestic design field in perceptual design[1].

\section{Sensibility and rationality in product design}

In terms of rational beauty of product design, Hegel thought that "rationality is the most complete cognitive ability, which is the advanced stage of cognition. Only rationality can reveal the essence of things." Of course, without exception for product design, the radical definition of design is the real meaning to solve the confronting problem.

In terms of the perceptual beauty of product design, the emotional beauty of products depends more on the subjective factors of people, to satisfy different inner feelings for people to meet their various needs. In the process of product design, if we can present or express the true emotional appeal in concise and clear way, and can arouse the emotional resonance of users through objective things, then the design will be successful. The beauty of single and rigorous reason is not powerful to

*Corresponding author's e-mail: 1789574764@qq.com 
give consumers warm (humane) feelings, and the beauty of unitary sensibility always forms excessive adornment and ignores the rationality of product function and structure easily.

Sensibility is the intuitive feeling of objective things and the emotional factors generated by the objective things acting on the senses, providing the basis for design. In the whole design process, the designer takes the perceptual cognition to the design, and then passes it to the applicable crowd through objective things. Sensibility serves as the bridge among product, user and designer. Rationality is the basis for a product to complete its work normally and efficiently. The two complements each other and provides important design basis for rationalization, emotionalization and humanization of product design. Considering sensibility and rationality in design at the same time, re-innovating the existing functions and appearance of products can improve the comprehensive performance of the product[2].

\section{Research on perceptual design in products}

In the design process, the most fundamental purpose of researching sensibility is to coordinate the relationship among people, people \&machines, people \&environment.
The sensibility and rationality in the design are intertwined, and the two ones are controlled properly for ensuring the quality of work. The product emotion is effectively passed to users to meet the expectations of designers, to satisfy users' emotional demands, for improving the user experience during the interaction between people\&product[3]. Generally speaking, sensibility is more diverse than rationality. With the trend of modern aesthetics, perceptual design must be multifunctional. People are expecting diversified and multifunctional products that can meet the various requests of users. But it is essential to choose appropriate functions. It will have a negative effect if a product owns too many functions even make it be bad quality and complicated processions.

In the process of product design, designers always need to know user feature and perceptual request of products. Combining with their own preference to complete product's specific features, the specific characteristics of the product, such as emotion, art, function, structure etc. are transformed according to the original product pain point. The generated products would pass the feeling and symbol to users, the receivers would reflect the different perceptual reactions, and designers will get feedbacks from the users at the same time, as shown in the below picture.

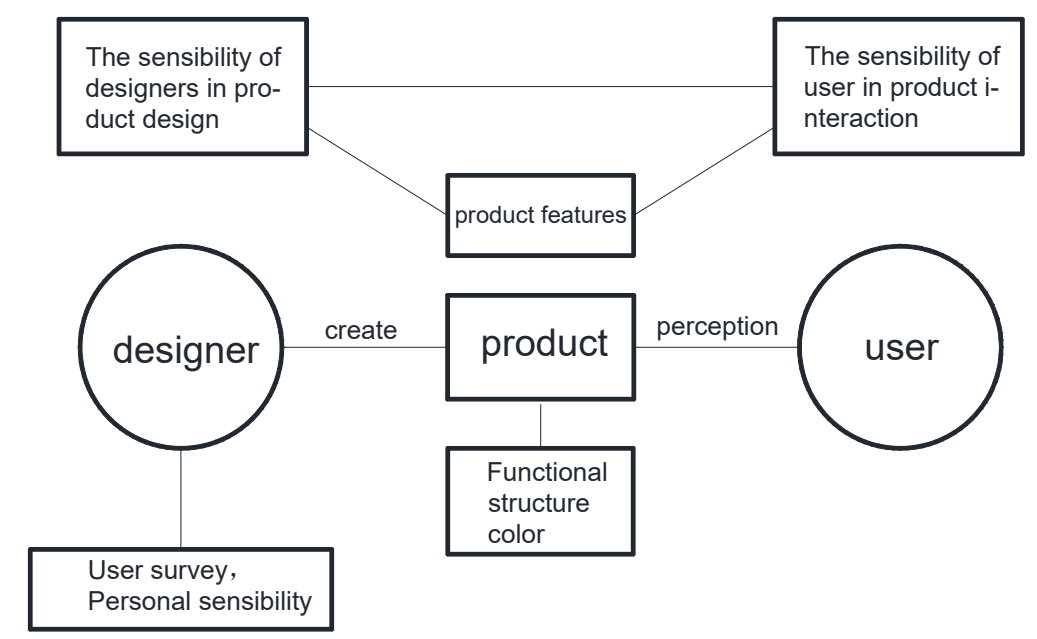

Figure 1. Flow chart of perceptual connection

\subsection{Research and analysis of perceptual design principles}

3.1.1. Perceptual design's directive to users. In the process of product interaction, the product is required to express its functions and usage methods to users, and a professor who is independent of himself can teach himself and speak for himself. Based on the product's shape and indicators (morphology, surface texture, color), it implies the use of product, so that users can use product independently[4].

You can express the direction as well as proportion through color or structural components that are combined and separated mutually first. With aesthetic functions, color is used to attract the user's attention and can indicate safety as well as prompt security, giving people different visual cues through vision.

The surface texture of product can convey certain information through the sense of touch. For example, with smooth surfaces, capable of reducing the design of edges and corners, children's toy products feel comfortable, thus bringing children a sense of security and comfort. The surface of mechanical products is rough and even unclear, with poor comfort, implying that the user is far away from the place. On the other hand, the texture can be treated separately on some components, such as setting appropriate stripe protrusions on the surface of handle, knob, etc. to increase friction. It is more suitable for operators. 


\begin{tabular}{|c|c|c|c|}
\hline & A & B & C \\
\hline 1 & Indicator sign & The function of hint. & Manifestation mode \\
\hline 2 & Form & Express different functions and operation modes. & Morphologic change \\
\hline 3 & Texture & Separate different structural components. & Smooth and rough \\
\hline 4 & Color & $\begin{array}{c}\text { Distinguish between different functional areas, } \\
\text { Indicates safety, indicates danger. }\end{array}$ & Color contrast \\
\hline
\end{tabular}

Figure 2. The hinting function and manifestation mode of indicator symbol

3.1.2. Emotional design conforms to the symbol of the product. Product cultural symbols include value symbols, aesthetic symbols, technology symbols, security symbols, etc. Emotional design is a unique way to satisfy people's spiritual requests for products. Beauty is what people desire for products, and aesthetic symbols run through the entire product design procession. Attributes pursued by any product and technical symbols require the external structure to express excellent quality of product, which visually makes people feel reliable, handling transitions between parts, surface texture, etc., and improving the grade of the product [5]. The security of mechanical and electric products is particularly important for all users. It not only needs profound technology, but also needs to give users a sense of physical, psychological security and trust in the appearance structure and color.

\subsubsection{Add perceptual factors in product design. It is} important to effectively extract the perceptual factors and symbols in the design procession. The extracted content must be representative and infectious in order to effectively express emotions. Otherwise, it only plays a pure decorative role in the increase of elements. On the contrary, it is a burden. The main evaluation criteria for product appearance factors (rationality, aesthetics, process ability and usability) are closely related to the product's perceptual experience [6]. Perceptual design reflects the combination of aesthetics as well as ergonomics, making the product more perceptual and more in line with modern aesthetic trends. It also puts forward higher requirements for designers, in terms of improving not only rational literacy, but also the depth of perceptiveness. Researches about it stimulate the sense of innovation and promote the development of perceptual design in product design.

\subsection{Analysis of perceptual design research methods}

3.2.1. Sensory interaction between product and human. The senses serve as the bridge between people and the outside world. They understand the objective world through vision, hear, smell, taste and touch, and generate subjective impressions. Then in the product design process, emotional elements can be incorporated into the design elements, so that the product can coordinate people's needs mutually, or provide a better user experience through a certain way to guide people's operation and spiritual impression, to make people produce some associations and cognition, thus breaking the stereotype.

Sensation is abstract. In the process of studying the characteristics of the senses, a certain medium is also needed to study the sensory characteristics of the senses, such as the visual study on sounds. It is not possible for people with hearing impairment to perceive sounds. I found two ways to help people perceive sound. The first one is to place some small particles on the vibrating acoustic surface. For different sound ranges, the small particles on the surface will pile up into different patterns, which helps people pass through vision. The sound is perceived intuitively, and the second is through sound wave images. The study on senses requires a certain medium. $80 \%$ of people's perception of the outside world comes from the research process of perceptual design in product design, like vision. The main research object has certain design requirements for products with different appearance shapes, textures, and colors. Thus, the formal beauty rule has become a research medium for perceptual design which is carried by the formal beauty rule in product design.

\subsubsection{Research strategy of perceptual design in} formal beauty. The application of formal beauty rules is a better way to apply perceptual design to product design. Researches on product modeling are one of the important factors in product design. To achieve a high level of artistic appearance, not only the three-dimensional composition methods and expression methods are studied, but also the characteristics of people's perception of the body are considered. For modern industrial products, on the basis of completing use functions of the product, it is necessary to step up the integration of material functions and its feasibility to well combine material functions and the spiritual functions it produced. That is to say, we must pay more attention to the aesthetics of the product's appearance, and affect the perception of people based on the appearance of the product. The perceptual design of product can start with the product form, deepen their impact on users, and use different forms to convey different emotions.

In the process of product design, the control and scale 
of proportion are the basis of product design. Of course, the complexity of product functions and the simplicity of operation directly affect the emotions of users. If you are willing to use a product or dislike it, the appearance of product will exert a more direct impact on the user's perception. Therefore, the question of proportion and scale play a crucial role in the perception design of the product. In the design and research phase, the scale and its issues must be considered by combing with ergonomics. Starting from the working process, perceptual design is carried out based on human data in terms of product operating components and working space, striving to give operators a better operating experience. Reasonable proportions as well as scales and the use of suitable human-machine data will closely connect the product with users, step up the coordination between the two, shorten the distance among the designer, the product, and the user, and contribute to emotional transmission and perceptual cognition.

Equilibrium and stability are also basic design requirements for product design, as well as the basis for the transmission of a sense of security, giving users more spiritual comfort. From a fundamental point of view, the product is composed of certain materials, masses and structural methods. It can be simply regarded as a reasonable stacking of components, and the product itself must have a certain sense of weight. Due to different materials, structural combination forms, proportions, and colors used by different products or different functional components of the same product, the users have different feeling of weight. The balance and stability of the product require the product to maintain a stable state in terms of the parts from the inside to the outside, and then convey the sense of balance to users.

Unity and change can create an atmosphere from the perspective of structure and color. As for the product design, it is necessary to achieve diversified artistic effects in the composition, and at the same time, the overall as well as local parts must be coordinated and unified. Different structural combinations and color combinations can be used on different products, or the same or different structures and color combinations can be used on the same product to achieve different visual effects. The users mainly perceive information of the outside world through vision. The unified and change processing method is the main way to perform perceptual design. Through the unified and change processing method, the product's emotional transmission to users can be easily performed.

\section{Conclusion}

As for the method research of perceptual design, it needs to focus on the users, conduct the users' experience survey based on the products available, so that we can get their potential disadvantage. Designers assign the perceptual elements into products, followed by passing various emotional issues to users via products. People's various knowledge basis can generate different reactions from the same perceptual point, which is essential to take the emotional response of most people as the standard.
Perceptual design was produced with the development of design scientific, society request, and mutual contribution of perception and rationality, both of which are essential design concepts for the complete product design. What's more, perceptual sensibility research provided basic theory support and technology channels. Perceptual design is more abstract compared with product design, and melting the perceptual design into the product design is a visualized process. It is necessary to take the beauty principle to be the bond to combine the perceptual design so that its emotionalization of product design can satisfy the emotional request for people when using the product. The More humanized impression is added to products made by perceptual design, and with the background of developing product design and growing product area, the perceptual design research is bound to catch more and more attention in the future.

\section{References}

1. Lixin Li (2006), engineering .The birth of a new discipline [J]. Art life. 73-75.

2. Chuyu Liu, Hailin Wang, Rong Chen(2002),Product design and evaluation method based on kansei engineering [J]. Packaging engineering.(10): 90-94.

3. Qi Yang(2014), on the perceptual characteristics of aesthetic activities $[\mathrm{J}]$. Northern literature (mid-ten-day issue),168-169.

4. Xuan Zheng, Yuxia $\mathrm{Hu}(2008)$, application of semiotics in product design $[\mathrm{J}]$, journal of hubei university of technology, 104-106.

5. Shuying Chen(2011), on the humanized design of industrial products $[\mathrm{J}]$, jintian, 72-72.

6. Yuzhong Shen(2007), research on positioning method of perceptual factors in product design [D],Journal of Shanghai jiao tong university. 